\title{
Age-period-cohort analysis of tuberculosis notifications in Hong Kong from 1961 to 2005
}

\author{
P Wu, ${ }^{1}$ B J Cowling, ${ }^{1}$ C M Schooling, ${ }^{1}$ I 0 L Wong, ${ }^{1} \mathrm{~J}$ M Johnston, ${ }^{1}$ C-C Leung, ${ }^{2}$ \\ C-M Tam, ${ }^{2}$ G M Leung ${ }^{1}$
}

- Additional data are published in the technical appendix and figs $1-3$ available online only at http://thorax.bmj.com/content/ vol63/issue4

${ }^{1}$ School of Public Health, Li Ka Shing Faculty of Medicine, The University of Hong Kong, Hong Kong, SAR, China; ${ }^{2}$ Tuberculosis and Chest Service, Department of Health, Government of the Hong Kong SAR, Hong Kong, SAR, China

Correspondence to:

Dr B J Cowling, School of Public Health, The University of Hong Kong, 21 Sassoon Road Pokfulam, Hong Kong, SAR,

China; bcowling@hku.hk

Received 4 April 2007 Accepted 9 October 2007

Published Online First 16 November 2007

\section{ABSTRACT}

Background: Despite its wealth, excellent vital indices and robust health care infrastructure, Hong Kong has a relatively high incidence of tuberculosis (TB) (85.4 per 100 000). Hong Kong residents have also experienced a very rapid and recent epidemiological transition; the population largely originated from migration by southern Chinese in the mid 20th century. Given the potentially long latency period of TB infection, an investigation was undertaken to determine the extent to which TB incidence rates reflect the population history and the impact of public health interventions.

Methods: An age-period-cohort model was used to break down the Hong Kong TB notification rates from 1961 to 2005 into the effects of age, calendar period and birth cohort.

Results: Analysis by age showed a consistent pattern across all the cohorts by year of birth, with a peak in the relative risk of TB at 20-24 years of age. Analysis by year of birth showed an increase in the relative risk of TB from 1880 to 1900 , stable risk until 1910, then a linear rate of decline from 1910 with an inflection point at 1990 for a steeper rate of decline. Period effects yielded only one inflection during the calendar years 1971-5.

Conclusions: Economic development, social change and the World Health Organisation's short-course directly observed therapy (DOTS) strategy have contributed to TB control in Hong Kong. The linear cohort effect until 1990 suggests that a relatively high, but slowly falling, incidence of TB in Hong Kong will continue into the next few decades.

Tuberculosis (TB) has been declared a global health emergency since 1993. ${ }^{1}$ Hong Kong, despite its wealth (GDP per capita was approximately US\$33 000 in 2005), excellent vital indices (longest life expectancies at birth for both sexes) and robust health care infrastructure, is classed as having an intermediate TB burden, ${ }^{2}$ with a notification rate for TB of all forms of 85.4 per 100000 population in 2006. While it is not facing the same critical situation as some south-east Asian and African countries, local notification rates are considerably higher than in most other developed countries. ${ }^{2}$ This apparent paradox of high wealth yet high burden may be due to the unique social history of Hong Kong where population growth has been driven by waves of immigration from southern China mainly after 1945, given that migration has been found to drive the incidence of TB in other industrialised countries. ${ }^{4-6}$

The age-period-cohort (APC) modelling framework ${ }^{7}$ has previously been used to study secular trends, typically in the incidence of chronic disease $^{89}$ but also in the incidence of infectious disease. ${ }^{10-13}$ The APC model breaks down the time trends in disease incidence into the effects of age, year of birth and calendar period. For instance, changes in risk by age can reflect pathogenesis of disease, while those by birth cohort can show the effect of prevalence of an infectious disease over a lifetime, and period effects can show changes in diagnostic efficiency. APC models have been applied to $\mathrm{TB}$ mortality, ${ }^{14}$ but we are not aware of previous applications to the incidence of $\mathrm{TB}$ While models for the incidence of infectious disease will typically describe transmission dynamics, these dynamics may be less important for $\mathrm{TB}$ given the chronic characteristics of infection, particularly the slow time course between infection and onset of symptoms and the high proportion of latent infections.

We have applied APC modelling techniques to TB notification rates in Hong Kong and interpret the results in terms of the impact of demographic shifts, socioeconomic development and intervention programmes on trends in the incidence of $\mathrm{TB}$.

\section{METHODS}

\section{Sources of data}

Age-specific and sex-specific TB notifications from 1961 to 2005 were obtained from the Department of Health of the Hong Kong Government. ${ }^{15}$ TB has been a notifiable infectious disease in Hong Kong since 1939 and notifications from the private and public sectors are centrally collated in the Department of Health. The annual age-specific and sex-specific mid-year populations of Hong Kong from 1961 to 2005 were derived from official statistics published by the Census and Statistics Department. ${ }^{16}$ Cases were notified if they met a clinical case definition or a laboratory case definition of active TB infection. ${ }^{17}$

\section{Statistical analysis}

Annual notification rates were standardised to the World Standard Population ${ }^{18}$ to investigate the changes in disease reporting rates over time, allowing for changes in the underlying population age structure.

In order to estimate the independent effects of age, period and birth cohort factors on $\mathrm{TB}$ notification, we fitted a negative binomial loglinear regression model ${ }^{19}$ with age, period and cohort terms included as categorical variables. We used a negative binomial rather than Poisson model to allow for potential overdispersion in the data due to clustering of cases. $^{20}$ Notifications were 
grouped into 185 -year age groups, 95 -year calendar periods and 26 birth cohorts. A unique feature of APC models is that the three components are interrelated, specifically any two factors allow the third to be derived. ' To overcome this identifiability problem, we constrained the second and penultimate period terms to be the reference groups. ${ }^{21}$

We compared the full APC model with three partial models adjusting for age, age-period and age-cohort terms, respectively. We further developed the APC model by including age-cohort interaction terms to investigate potential heterogeneities in age effects in different groups of birth cohorts, as described further in the technical appendix available online. Alternative models were compared using the Akaike information criterion (AIC), which is a measure of goodness of fit adjusting for model parsimony where a lower value of AIC indicates a better model. ${ }^{22}$ The AIC is derived as -2 multiplied by the fitted loglikelihood plus twice the number of parameters included in the model. In addition, three of the authors independently assessed residual plots by visual inspection to verify the goodness of fit of the regression models. The Pearson residuals for the regression model were calculated as the standardised differences between the observed and fitted values; further details are given in the online technical appendix. All statistical analyses were performed using $\mathrm{R}$ version 2.4.1 (R Development Core Team, Vienna, Austria).

\section{RESULTS}

Figure 1 shows declining trends in age- and sex-standardised TB notification rates by sex from 1961 to 2005. Disease reporting rates have mostly levelled off in the last decade compared with the more rapid decrease in earlier years. Annual age- and sexstandardised rates fell 83\% from 405 to 67 cases per 100000 during the observation period. Notification rates have declined more rapidly in men than in women, but notification rates in men currently remain approximately $70 \%$ higher than in women. The apparent spike in 1967 is most likely an artefact due to a reorganisation of TB statistics in that year. ${ }^{23}$ Agespecific notification rates have historically been much higher in men, and particularly in older men, than in women (see fig 1 in online appendix). In both sexes TB notifications typically rose with age to a mode in the early adult years, then declined through adult life before increasing again with older age. However, this pattern has become less distinct, with more recent years recording far lower rates in younger individuals, but similar notification rates in those over 75 years of age.

We fitted age, age-period, age-cohort and age-period-cohort models to the incidence rates and found that, among these, the

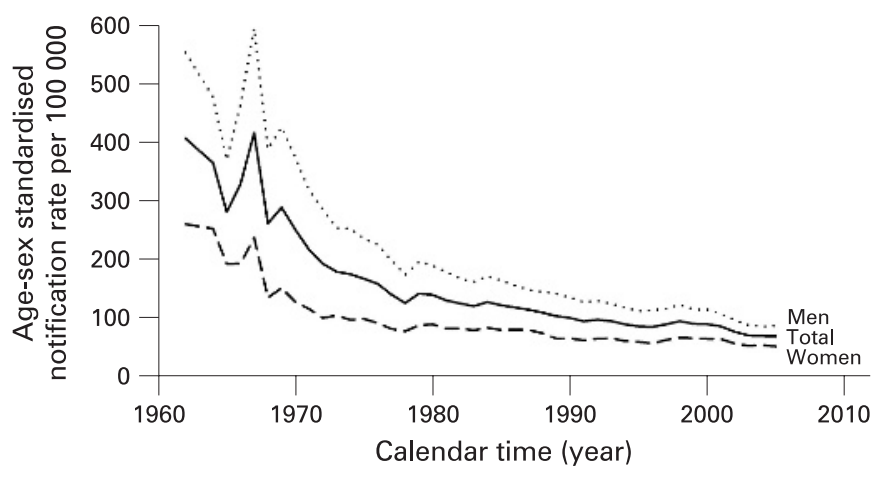

Figure 1 Age- and age-sex standardised TB notification rates in Hong Kong, 1961-2005. Standardised to the WHO World Standard Population. full APC model was the most appropriate for describing the data for both sexes since it had the lowest AIC. However, examination of residual plots revealed that the full APC model was not optimal statistically; the fit was improved considerably after inclusion of age-cohort interaction terms. We investigated a variety of categorisations of the age groups and birth cohorts and found that allowing for separate age effects in each of three groups of birth cohorts divided at 1906 and 1956 resulted in the best fitting model - that is, the model which most accurately represented the raw data according to the AIC and visual inspection of residual plots.

Figure 2 presents the age, period and cohort parameter estimates after adding the interaction term between age and cohort into the model. Figures $2 \mathrm{~A}$ and $2 \mathrm{D}$ show that people born in the three birth cohort groupings had similar changes in risk of $\mathrm{TB}$ with age, noting that the age effects for the most recent birth cohort group were shifted upwards relative to the age effects for the other two birth cohort groups as a result of the choice of the reference groups, while the corresponding cohort effects for the birth cohorts after 1956 are shifted downwards by a similar factor. Adjusting for the effects of birth cohort and time period, the relative risk of age began to rise in both males and females in the 5-9 year age group, peaked at 2024 years, then quickly declined to a nadir in the $45-49$ year age group, and finally showed an upturn during the eighth decade for women which was not apparent for men. In both sexes the risk of TB showed a turning point at approximately 1906 as the central year of birth, and another second-order change appeared in the 1991 birth cohort. Period effects in men and women yielded only one inflection during the calendar years 1971-5. The overall patterns in the APC parameter estimates were similar across the sexes. Whereas both sexes (female more so than male) showed an upward inflection as they approached their 70s, this effect was only observed for people born later (1911-56) but not earlier (1876-1906). Finally, there may be an additional discontinuity or second-order change in the immediate post-war period around 1950 which is more pronounced for men than women, although this may be an artefact because it coincides with the birth cohort strata cut-off.

\section{DISCUSSION}

We have described a novel application of APC modelling to studying trends in TB incidence. While APC models are typically applied to study trends in chronic diseases, ${ }^{8}$ they may also be used to analyse infectious diseases. ${ }^{10-13}$ Given the long time scale of the TB infection process, the majority of infections being latent, and the likely huge pool of infected individuals at least among earlier generations, ${ }^{24}$ it is arguable that $\mathrm{TB}$-or at least reactivation disease which forms the majority of TB notifications in Hong Kong (particularly in the elderly) ${ }^{25}{ }^{26}$ - could be considered more of a chronic condition than an infectious disease. Therefore, although our model does not explicitly incorporate the infection processes or transmission dynamics of TB in a population, it can still allow us to make inferences on trends in disease incidence. While APC models are typically based on the Poisson distribution, implying an underlying assumption of independence between events, we have used the negative binomial distribution to allow for the possibility of clustered events. ${ }^{20}$ Our results will provide a useful and informative complement to more detailed future analyses with complex transmission models.

Our results have revealed important temporal shifts in the aetiology of $\mathrm{TB}$ in Hong Kong that can be explained by demographic change, intergenerational effects and historical 

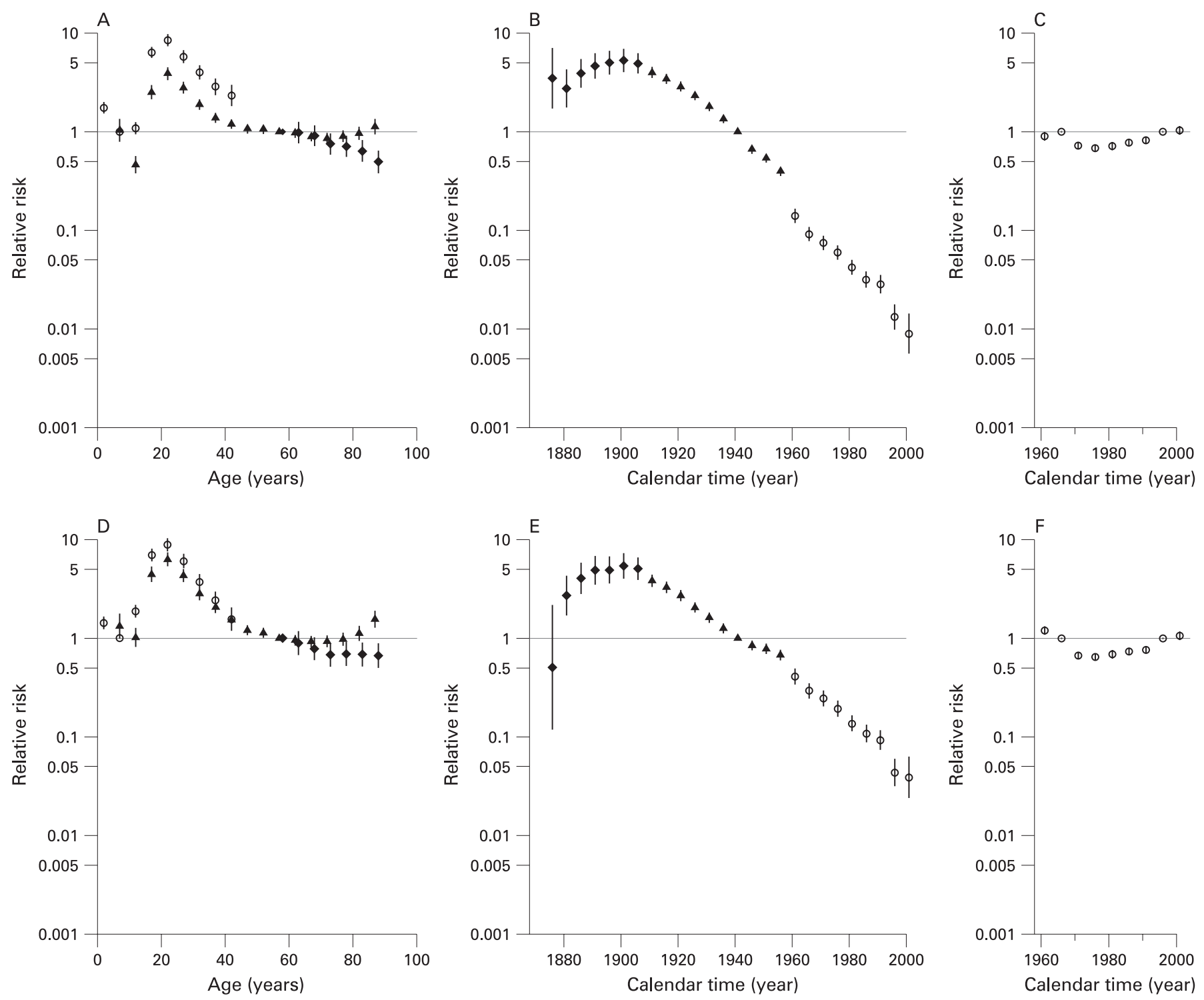

Figure 2 Parameter estimates of age, period and cohort effects from the full APC model with age-cohort interaction term for men (above) and women (below). (A) Estimated age effects (circles for people born after 1961, triangles for people born in 1911-56, diamonds for people born in 1876-1906) with $95 \%$ confidence intervals for men. (B) Estimated cohort effects (circles for people born after 1961, triangles for people born in 1911-56, diamonds for people born in 1876-1906) with 95\% confidence intervals for men. (C) Estimated period effects (circles) with 95\% confidence intervals for men. (D) Estimated age effects (circles for people born after 1961, triangles for people born in 1911-56, diamonds for people born in 1876-1906) with 95\% confidence intervals for women. (E) Estimated cohort effects (circles for people born after 1961, triangles for people born in 1911-56, diamonds for people born in 1876-1906) with 95\% confidence intervals for women. (F) Estimated period effects (circles) with $95 \%$ confidence intervals for women.

events. One of the most vexed local public health issues is whether our currently high TB burden is mainly being driven by primary infections, reinfections or reactivations. Since the global resurgence of TB in the 1980s, more studies have focused on the impact of recurrence (reinfection and reactivation) on $\mathrm{TB}$ epidemics. ${ }^{27-29}$ We cannot directly examine the relative influences of reactivation or reinfection versus primary infection since our dataset only describes aggregate disease notifications and we do not have information on how many of the notifications are reactivations of previous infections. However, while our crude data suggest that the risk of active TB increases with older age in the over 50s (see fig 1 in online appendix), the results of our analysis indicate that the increased risk may be attributed to earlier birth cohorts rather than more advanced age per se, except in the "old-old" and with increasing longevity (fig 2). This implies that earlier generations of migrants were already infected in mainland China when they were young before settling in Hong Kong. Many had reactivation diseases during later adulthood which were recorded in the local notification system. Since the proportion of mainland migrants had shrunk continuously during the period of observation (fig 2 in online appendix), and while TB rates have come down with economic development and population level intervention measures in the more prosperous parts of China such as Guangdong province ${ }^{30}$ from where most of the migrants to Hong Kong originated, we can predict that there will be fewer reactivation cases and the overall incidence of TB in Hong Kong will continue to fall.

A number of studies have shown that migrants-specifically from developing countries with a higher prevalence of $\mathrm{TB}$-are affecting the trends of TB incidence in developed countries. ${ }^{4}{ }_{31}$ In China, migrant workers from rural areas-who comprise $10 \%$ 
of the country's population-joining the wage economy in towns and cities form an important vector for TB spread and the maintenance of a high burden in economically developed urban regions. ${ }^{32}$ While only a small percentage of the TB notifications in Hong Kong are from recent migrants, ${ }^{23}$ Hong Kong has a unique social history where the local population has historically been augmented by waves of immigrants from mainland China in the 20th century, mainly in the late 1940s, early 1950s and the late 1970s (see fig 2 in online appendix) who then subsequently developed reactivation disease locally. ${ }^{33}$ About one million people migrated into Hong Kong in the late 1940s and early 1950s, largely young people looking for work, but also around 1949 some political refugees from Guangzhou and to a much lesser extent Shanghai. ${ }^{34}$ Hong Kong birth cohorts born before 1945 are largely (approximately 80\%) migrants from southern China, while birth cohorts born after 1960 are largely Hong Kong born (see fig 3 in online appendix). ${ }^{35}$ Thus, the turning point in the cohort effect around 1910 is likely to reflect events in southern China rather than specifically in Hong Kong. It is tempting to interpret the upstroke of the turning point as a population-wide embodiment of the turmoil connected with the fall of the Oing dynasty, driven by increased population movement and exposure for the cohort of babies born around that time. However, the downstroke of the inflection might be an artefact of the major wave of migration into Hong Kong in the late 1940s of people in their 20s and 30s. Older migrants at that time from the pre-1906 birth cohorts would have been aged $\geqslant 40$ years and most likely to be political refrgees from a social stratum with generally better living conditions than migrant workers, and therefore may have been less exposed to TB in early life. Moreover, any older migrants world, by definition, have been strongly selected healthy survivors at a time when life expectancy in China was $<40$ years.

On the other hand, given the difference in living standards between China and Hong Kong, it is noteworthy that there is no obvious downward inflection in the post-1945 birth cohorts until possibly about 1990, even though an increasingly higher proportion were born and grew up in Hong Kong with its rapidly developing economy and elements of a modern infrastructure (sanitation and public health initiatives). There are several possible explanations for this lack of downward inflection in the earlier cohort. First, population mixing engendered by migration may have introduced a large pool of susceptible individuals from rural areas where TB was less prevalent than in urban areas, presumably due to the reduced exposure in such isolated closed communities. ${ }^{36}$ This enhanced circulation of TB may have counteracted improved living standards and public health initiatives. Second, living standards in China were essentially unchanged from pre-industrial levels until well into the second half of the 20th century, so older migrants (corresponding to earlier birth cohorts) would have had a cumulatively greater exposure to pre-industrial living conditions, and it may be that it is the cumulative exposure to poor living conditions rather than exposure at a critical period (eg, during growth and development) which is important for $\mathrm{TB}$, either primary infection or recurrence. Third, it is possible that because of the dynamics of TB infection there is a long lag between better living conditions and a reduction in $\mathrm{TB}$, so that several decades of improvements in living conditions are needed before the prevalence in the population falls to a level which precludes widespread infection. Certainly, the finding that even in the birth cohort from around 1960 about 80\% were tuberculin positive at primary school, strongly suggests widespread circulation of TB in birth cohorts up to that date and such a time lag. Only by about the 1980 birth cohort did the proportion of tuberculin positive fall to $20 \%,{ }^{37}$ suggesting that it took about a generation of improved living conditions and BCG vaccination for the level of TB circulation to fall dramatically. The sharp decrease for the most recent birth cohorts may be a reflection of the reduced circulation of and exposure to TB in these more recent cohorts. The implication for the future is that slowly declining levels of TB are very likely in older people in the short to medium term, after which there may be a more substantial fall.

Our data do not go back far enough in time to capture any potential period effect of newborn BCG vaccination since 1952. Nevertheless, there is substantial evidence in the literature to suggest that BCG probably does not change incidence patterns but can prevent serious complications and reduce paediatric mortality, especially in countries with a high TB prevalence. ${ }^{38}$ Empirically, based on local observations, the introduction of the BCG programme has resulted in a dramatic decline in infant mortality due to $\mathrm{TB}^{39}$ and a concomitant reduction in the risk of infection for young children, ${ }^{37}$ but there is still no conclusive evidence for the benefit of BCG vaccination to protect adults from infection. ${ }^{40}{ }^{41}$

The effective treatment of infected patients appears to be a much more important determinant of overall incidence. Our period inflection point centres around the 1970s, coinciding with the introduction of directly observed therapy (DOT) in 1970 which progressed to the incorporation of the short-course regimen (DOTS) in 1979. ${ }^{37}$ This is consistent with demonstration of the benefits of DOTS elsewhere. ${ }^{42}$ In addition to the development of effective medical interventions, societal changes could be another possible explanation for the declining incidence of $\mathrm{TB}$ in Hong Kong. The average household size (thus density, assuming constant or increasing living space) in Hong Kong has been decreasing for decades, which might to some extent have prevented TB spread by reducing airborne dissemination.

Treating potentially infected individuals should be another focus of TB control systems in developed regions with a relatively lower incidence of $\mathrm{TB}$. However, while treating latent $\mathrm{TB}$ infection is likely to speed the decline in the incidence of $\mathrm{TB}$, it might prove costly as the effectiveness of chemoprophylaxis requires accurate diagnoses, high treatment adherence and minimisation of potential adverse drug reactions. Further study is therefore needed to examine the potential cost-effectiveness of such a control strategy.

The considerable sex differences in $\mathrm{TB}$ incidence rates in Hong Kong shown in fig 1 is unlikely to be associated either with HIV (because of a low positive rate of HIV among TB patients ${ }^{23}$ ) or sex-based inequality in accessing services given Hong Kong's free TB clinics and universal health care and the fact that women have less serious symptoms than men at the time of diagnosis. ${ }^{43}$ Recent studies on the association between smoking and TB in Hong Kong found that the much higher prevalence of smoking in men accounted for approximately $45 \%$ of the sex difference. ${ }^{44} 45$

Increasing longevity over time and survival to a very old age (favouring women over men and later cohorts over earlier cohorts) together explain the age-specific notification patterns historically (fig 1 in online appendix) and the age component results on breakdown analysis (fig 2 ). At the other age extreme, the peak risk during adolescence might have resulted from weaker immunity during growth spurts, ${ }^{46}$ increased public activities leading to more mixing or initiation of tobacco use. ${ }^{47} 48$ 
In conclusion, our results highlight the sex differential in the incidence of $\mathrm{TB}$ and the continuing peak in disease risk for young adults. The epidemiological transition experienced by Hong Kong residents with migration from China, DOTS and increased longevity and survival have probably been the most important determinants of TB notification rates in Hong Kong. Nevertheless, there remains an intermediate burden which is most likely due to a lead time before these public health improvements could have had a significant impact on the circulation of $\mathrm{TB}$ and to the reactivation of $\mathrm{TB}$ in people originally infected under very different circumstances. As a result, it is likely that a relatively high but predictably falling burden of TB in Hong Kong will continue into the coming decades, assuming no major changes to immigration policies.

Acknowledgements: The authors thank Ms Marie Chi for secretarial support.

Funding: This work was supported in part through a commissioned research grant from the Research Fund for the Control of Infectious Diseases of the Health, Welfare, and Food Bureau of the Hong Kong Special Administrative Region Government.

Competing interests: None.

\section{REFERENCES}

1. World Health Assembly. Tuberculosis programme. WHA46.36. Geneva: World Health Organization, 1993

2. World Health Organization. Global tuberculosis control - surveillance, planning, financing. Geneva: World Health Organization, 2006.

3. Department of Health Center for Health Protection. Notification and death rate of tuberculosis (all forms), 1947-2006. Hong Kong: The Government of Hong Kong SAR, 2006.

4. Farah MG, Meyer HE, Selmer R, et al. Long-term risk of tuberculosis among immigrants in Norway. Int J Epidemiol 2005;34:1005-11.

5. Lillebaek T, Andersen AB, Bauer J, et al. Risk of Mycobacterium tuberculosis transmission in a low-incidence country due to immigration from high-incidence areas. J Clin Microbiol 2001;39:855-61.

6. Borgdorff MW, Behr MA, Nagelkerke NJ, et al. Transmission of tuberculosis in San Francisco and its association with immigration and ethnicity. Int J Tuberc Lung Dis 2000:4:287-94.

7. Holford TR. Understanding the effects of age, period, and cohort on incidence and mortality rates. Annu Rev Public Health 1991;12:425-57.

8. Clayton D, Schifflers E. Models for temporal variation in cancer rates II: Age-periodcohort models. Stat Med 1987;6:469-81.

9. Wong IOL, Cowling BJ, Schooling CM, et al. Age-period-cohort projections of breast cancer incidence in a rapidly transitioning Chinese population. Int $J$ Cancer 2007; 121:1556-63.

10. Verdecchia A, Mariotto A, Capocaccia R, et al. An age and period reconstruction of the HIV epidemic in Italy. Int J Epidemiol 1994;23:1027-39.

11. Préziosi M-P, Yam A, Wassilak SGF, et al. Epidemiology of pertussis in a West African community before and after introduction of a widespread vaccination program. Am J Epidemiol 2002;155:891-6.

12. Kongsomboon K, Singhasivanon P, Kaewkungwal J, et al. Temporal trends of dengue fever/dengue hemorrhagic fever in Bangkok, Thailand from 1981 to 2000: an age-periodcohort analysis. Southeast Asian J Trop Med Public Health 2004;35:913-7.

13. Houweling H, Wiessing L, Hamers F, et al. An age-period-cohort analysis of 50,875 AIDS cases among injecting drug users in Europe. Int J Epidemiol 1999:28:1141-8.

14. Collins JJ. The contribution of medical measures to the decline of mortality from respiratory tuberculosis: an age-period-cohort model. Demography 1982;19:409-27.

15. Centre for Health Protection. Statistics on communicable diseases - notifiable infectious diseases. Hong Kong: Department of Health of the Government of the Hong Kong SAR http://www.chp.gov.hk/

notifiable1.asp?lang $=$ enfid $=43$ qpid $=26$ gppid $=10$ (accessed 7 August 2007)

16. Census and Statistics Department. Population by age group and sex. Hong Kong: Census and Statistics Department of the Government of Hong Kong SAR, 2006.

17. Hong Kong Tuberculosis and Chest Services. Guidance notes for notification of tuberculosis. Hong Kong: Department of Health of the Government of Hong Kong SAR, 2001
18. Ahmad OB, Boschi-Pinto C, Lopez AD, et al. Age standardization of rates: a new WHO standard. GPE Discussion Paper No. 31. Geneva: World Health Organisation, 2001.

19. McCullagh P, Nelder JA. Generalized linear models. 2nd ed. London: Chapman and Hall, 1989 .

20. Eggenberger F, Polya G. [Über die Statistik Verketteter Vorgänge. Zeitschrift für Angerwandte Mathematik and Mechanik.] J Appl Math Mechan 1923;1:279-89.

21. Robertson C, Boyle P. Age-period-cohort analysis of chronic disease rates. I: Modelling approach. Stat Med 1998:17:1305-23.

22. Burnham KP, Anderson DR. Model selection and multimodel inference: a practicaltheoretic approach. 2nd ed. New York: Springer-Verlag, 2002.

23. Hong Kong Tuberculosis and Chest Services. Annual report. Hong Kong: Department of Health of the Government of Hong Kong SAR, 2004.

24. Dye C, Scheele S, Dolin P, et al. Consensus statement: global burden of tuberculosis: estimated incidence, prevalence, and mortality by country. WHO Global Surveillance and Monitoring Project. JAMA 1999;282:677-86.

25. Chan-Yeung M, Tam CM, Wong H, et al. Molecular and conventional epidemiology of tuberculosis in Hong Kong: a population-based prospective study. J Clin Microbiol 2003:41:2706-8.

26. Vynnycky E, Borgdorff MW, Leung CC, et al. Limited impact of tuberculosis control in Hong Kong: attributable to high risks of reactivation disease. Epidemiol Infect 3 Aug 2007;1-10 (Epub ahead of print).

27. Le HO, Davidson PT. Reactivation and exogenous reinfection: their relative roles in the pathogenesis of tuberculosis. Curr Clin Top Infect Dis 1996;16:260-76.

28. Lambert ML, Hasker E, Van Deun A, et al. Recurrence in tuberculosis: relapse or reinfection? Lancet Infect Dis 2003:3:282-7.

29. Jasmer RM, Bozeman L, Schwartzman K, et al. Recurrent tuberculosis in the United States and Canada: relapse or reinfection? Am J Respir Crit Care Med 2004;170:1360-6.

30. Liu JJ, Yao HY, Liu EY. Analysis of factors affecting the epidemiology of tuberculosis in China. Int J Tuberc Lung Dis 2005;9:450-4.

31. Baussano I, Bugiani M, Gregori D, et al. Impact of immigration and HIV infection on tuberculosis incidence in an area of low tuberculosis prevalence. Epidemiol Infect 2006;134:1353-9

32. Wang L, Liu J, Chin D. Progress in tuberculosis control and the evolving public-health system in China. Lancet 2007;369:691-6.

33. Tsang S. A modern history of Hong Kong. Hong Kong: Hong Kong University Press 2004.

34. Hong Kong Government. Hong Kong annual report, 1954. Hong Kong: Hong Kong Government, 1955.

35. Hong Kong Census and Statistics Department. 1996 Population By-census: main report. Hong Kong: The Government of Hong Kong SAR, 1997.

36. Hou Y. Chinese demographic history. Shanghai: Fudan University Press, 2001.

37. Centre for Health Protection. Tuberculosis control in Hong Kong. Hong Kong: Department of Health of the Government of Hong Kong SAR, 2000.

38. World Health Organization. Guidance for national tuberculosis programmes on the management of tuberculosis in children. Geneva: World Health Organization, 2006.

39. Yeung CY. Evolution of child health care in Hong Kong. Hong Kong J Paediatr 2001;6:66-71.

40. Brewer TF, Colditz GA. Relationship between bacille Calmette-Guérin (BCG) strains and the efficacy of BCG vaccine in the prevention of tuberculosis. Clin Infect Dis 1995:20:126-35.

41. Colditz GA, Brewer TF. Efficacy of BCG vaccine in the prevention of tuberculosis. JAMA 1994;271:698-702

42. Dye C, Garnett GP, Sleeman K, et al. Prospects for worldwide tuberculosis contro under the WHO DOTS strategy. Lancet 1998;352:1886-91.

43. Chan-Yeung M, Noertjojo K, Chan SL, et al. Sex differences in tuberculosis in Hong Kong. Int J Tuberc Lung Dis 2002;6:11-8.

44. Leung CC, Yew WW, Chan CK, et al. Smoking and tuberculosis in Hong Kong. Int J Tuberc Lung Dis 2003:7:980-6.

45. Leung CC, Li T, Lam TH, et al. Smoking and tuberculosis among the elderly in Hong Kong. Am J Respir Crit Care Med 2004:170:1027-33.

46. Coberly JS, Comstock GW. Epidemiology of tuberculosis. In: Raviglione MC, ed. Reichman and Hershfield's tuberculosis: a comprehensive, international approach. New York: Informa Healthcare USA, 2006:65-92.

47. Altet N, Alcaide J, Lozano P, et al. Smoking as risk factor of tuberculosis in children and youth. Tuber Lung Dis 1994;75(Suppl 1):68-9.

48. Levy MH, Connolly MA, O'Brien RJ. Cigarette smoking as a risk factor for tuberculosis in young adults: a case-control study. Tuber Lung Dis 1996:77:570. 